\title{
Super-sniffer mice put their noses to the grindstone
}

The ability to smell arises from an intricate system of olfactory sensory neurons in the nose that transduce and encode information about the outside chemical environment. Odors bind to specific protein receptors, or odorant receptors (ORs), expressed by olfactory sensory neurons, kicking-off the complex process that ultimately leads to the perception of smell. Despite having over 1,200 different OR genes to choose from, individual olfactory sensory neurons express only one type of OR. However, the manner in which these neurons 'choose' which type of OR to express has been a longstanding question in developmental neurobiology.

A recent report by D'Hulst and colleagues (Cell Rep. doi: 10.1016/j.celrep.2016.06.047, published online July 7,2016 ) presents a new method for biasing large amounts of mouse olfactory sensory cells to express a specific type of OR. In addition to providing a platform for understanding the mechanisms of receptor expression in vivo, a fascinating by-product of the research is

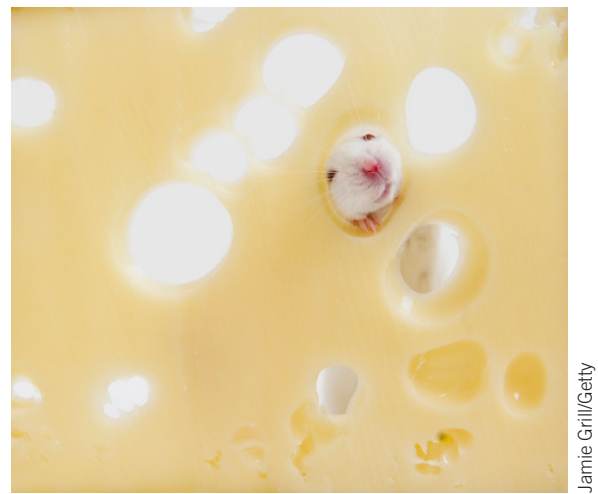

the creation of super-smelling mice, which the scientists call 'MouSensors'.

To generate these MouSensors, the authors first developed a transgenic strategy using DNA vectors of short 'enhancer elements' to increase the probability of expression of specific ORs within olfactory neurons. Based on previous work the group had conducted, they chose an enhancer element of a specific ORM71-to test their new transgenic strategy.
Using real-time qPCR and imaging methods, they found that their modified mice had significantly higher proportions of olfactory neurons expressing M71; just as they had anticipated. In vivo functional imaging showed that the increase of M71 expressing neurons was accompanied by stronger activation in the olfactory bulb in response to M71-specific odor stimuli. Additionally, the mice showed enhanced behavioral sensitivity to these specific odors, based on results from an olfactory discrimination task.

These results have some potentially interesting and important practical applications. D'Hulst et al. propose using these MouSensors, and possibly other animal species manipulated with their technology, to overexpress ORs that might allow animals to detect important scents at extremely low concentrations. This translational research could lead to improved bomb-sniffing animals, and even animals that can detect and 'diagnose' various diseases.

James E. Niemeyer 